\title{
Magic Labelings on Cycles and Wheels
}

\author{
Andrew Baker and Joe Sawada \\ University of Guelph, Guelph, Ontario, Canada, N1G 2W1 \\ \{abaker04, jsawada\}@uoguelph.ca
}

\begin{abstract}
We present efficient algorithms to generate all edge-magic and vertex-magic total labelings on cycles, and all vertex-magic total labelings on wheels. Using these algorithms, we extend the enumeration of the total labelings on these classes of graphs.
\end{abstract}

\section{Introduction}

Consider a wireless network in which every device must be able to connect to a subset of the other devices in the network using a unique channel to prevent collisions. One way to create such a channel assignment is to give numeric labels to the devices and channels in such a way that the labels of two devices and the communication line between them sum to a consistent value across every pair of devices in the network. In this case, knowing the labels of the two communicating devices gives the identification number of the communication line between them $[1]$.

This solution is an example of an edge-magic total labeling (EMTL). EMTLs are one application of the "magic" concept of magic squares to graphs. Given a simple undirected graph $G=(V, E)$, let $\lambda$ be a mapping from the numbers 1 through $|V|+|E|$ to the elements (vertices and edges) of $G$ such that each element has a unique label. An edge-magic total labeling is a labeling $\lambda$ in which the weight of each edge is the same. The weight of an edge is obtained by the sum of the label of the edge and the labels of its two endpoints and denoted by $w(e)$. If the weight is the same for every edge, it is termed the magic constant of the labeling, and is given by $h$. For an example of an EMTL with $h=20$, see Fig 1(a).

A vertex-magic total labelling (VMTL) is a labeling $\lambda$ in which the weight $w(v)$ of each vertex is the same. The weight of a vertex is obtained by adding the sum of the labels of the incident edges to the label of the vertex itself. If the weight is the same for every vertex in the graph, it is called the magic constant and is given by $k$. For an example of an VMTL with $k=20$, see Fig 1(b).

A totally magic labeling is a labeling $\lambda$ which is simultaneously both a vertexmagic total labeling and an edge-magic total labeling. The magic constants $h$ and $k$ are not necessarily equal. The class of totally magic graphs (those which admit a totally magic labeling) is much more restricted than the edge-magic or vertexmagic graphs. Figure 2 gives an example of a totally magic labeling on the cycle $C_{3}$. The only known connected totally magic graphs are $K_{1}, K_{3}$, and $P_{3}$. There 
a)

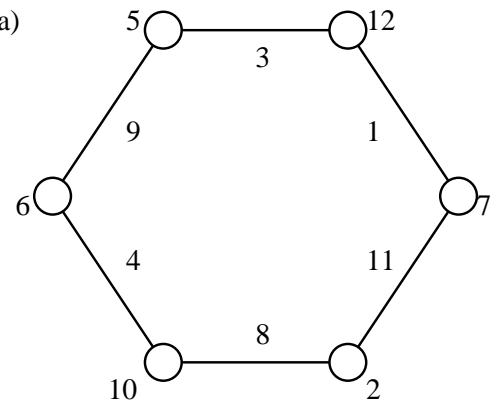

b)

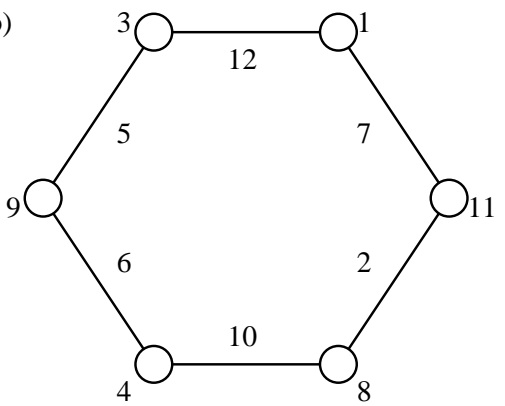

Fig. 1. Two $C_{6}$ graphs with corresponding edge-magic and vertex-magic total labelings. a) gives an edge-magic total labeling, and b) gives a vertex-magic total labeling.

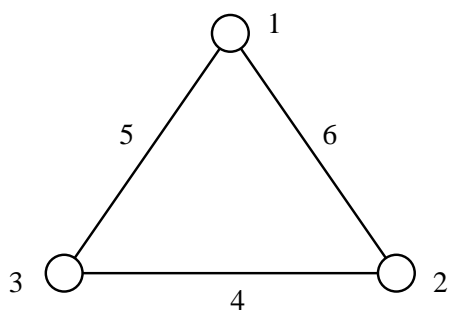

Fig. 2. The graph $C_{3}$ with a totally magic labeling. In this case, $h=9$ (the edge-magic constant), and $k=12$ (the vertex-magic constant).

are however an infinite number of disconnected totally magic graphs, as any graph consisting of a union of $2 n+1(n \geq 0, n \in \mathbb{Z})$ disjoint triangles is a totally magic graph [1]. There are additional types of magic labelings described beyond EMTLs, VMTLs and totally magic labelings. For a more complete treatment, see Gallian's dynamic survey [2].

Depending on which labels are assigned to vertices and which to edges, it is possible to achieve labelings with different magic constants on the same graph. A lower bound for a VMTL is obtained by applying the largest $|V|$ labels to the vertices, while an upper bound is found by applying the smallest $|V|$ labels to the vertices. Summing the weights of every vertex in a VMTL gives us $\sum_{v \in V} w(v)=$ $|V| k$. Every vertex label contributes to one weight (the weight of that vertex) while every edge label contributes to two weights (the weights of its two end points). Thus $|V| k=\sum_{v \in V} \lambda(v)+2 \sum_{e \in E} \lambda(e)$. By applying either the $|V|$ smallest or largest labels to the vertices, we can obtain the inequality

$$
\frac{13 n^{2}+11 n+2}{2(n+1)} \leq k \leq \frac{17 n^{2}+15 n+2}{2(n+1)}
$$

which gives basic limits on the magic constant of a graph without taking into account the structure of the graph [3]. Once the structure of the graph is taken 
into account, additional limits may be found. The set of integers which are delimited by these upper and lower bounds is the feasible range. The values which are the magic constant for some VMTL of a graph form the graph's spectrum. Therefore the spectrum is a subset of the feasible range.

In this paper we focus on finding all non-isomorphic VMTLs for cycles and wheels. Section 2 presents previous results with respect to vertex-magic total labelings on cycles and wheels. Sections 3 and 4 detail the enumeration algorithms and results for cycles and wheels respectively. Open problems for further research are presented in Section 5 .

\section{Background}

Throughout this paper, we focus primarily on two classes of graph, the cycles and the wheels. The cycle $C_{n}$ is given by the vertex set $v_{1}, v_{2}, \ldots, v_{n} \in V(G)$, and edge set $e_{i} \in E(G)$ where for $1 \leq i<n, e_{i}=\left\{v_{i}, v_{i+1}\right\}$ and $e_{n}=\left\{v_{1}, v_{n}\right\}$. Cycles are regular graphs (graphs in which every vertex has the same degree) as every vertex has degree 2 . The wheels $W_{n}$ consist of a cycle $C_{n}$ together with an additional dominating vertex. A dominating vertex is a vertex which is adjacent to every other vertex in the graph. Figure 3 shows a sample wheel graph $\left(W_{6}\right)$ and illustrates the naming scheme we will use while discussing parts of a wheel. Except for $W_{3}$, the wheels are not regular graphs.

\subsection{Cycles}

Every vertex-magic total labeling on a cycle (and indeed any regular graph) has a mirrored dual labeling. This property allows us, given an original labeling $\lambda$ on graph $G$, to obtain the dual labeling $\lambda^{\prime}$ given by $\lambda^{\prime}(v)=|V|+|E|+1-\lambda(v)$ for all vertices $v \in V(G)$ and $\lambda^{\prime}(e)=|V|+|E|+1-\lambda(e)$ for all edges $e \in E(G)$. The resulting magic constant $k^{\prime}$ is given by $k^{\prime}=6 n+3-k$ for cycles [4]. Consequently, the distribution of VMTLs by magic constant is symmetrical over the feasible range, and the presence of a VMTL achieving a magic constant in the upper half of the feasible range may be inferred by the presence of the dual labeling achieving the corresponding magic constant in the lower half of the feasible range.

Cycles also have a one-to-one correspondence between their edge- and vertexmagic total labelings. To obtain an EMTL $\lambda_{e}$ from a vertex-magic total labeling $\lambda_{v}$, set $\lambda_{e}\left(v_{i}\right)=\lambda_{v}\left(e_{i}\right)$ and $\lambda_{e}\left(e_{i}\right)=\lambda_{v}\left(v_{(i+1) \bmod |V|}\right)$ [4]. Figure 1 shows this correspondence graphically. Due to this relationship with EMTLs (which were developed earlier than VMTLs), previous work has been done to enumerate the edge-magic (and therefore also the vertex-magic) total labelings on cycles. The cycles $C_{3}$ through $C_{10}$ were completely enumerated by Godbold and Slater [5]. We confirm these calculations, and also count the number of VMTLs/EMTLs on the cycles $C_{11}$ through $C_{18}$.

Godbold and Slater show that a VMTL exists for every feasible magic constant for $C_{n}$ when $n>4$ [5]. Our enumeration breaks down the results for cycles by magic constant. 


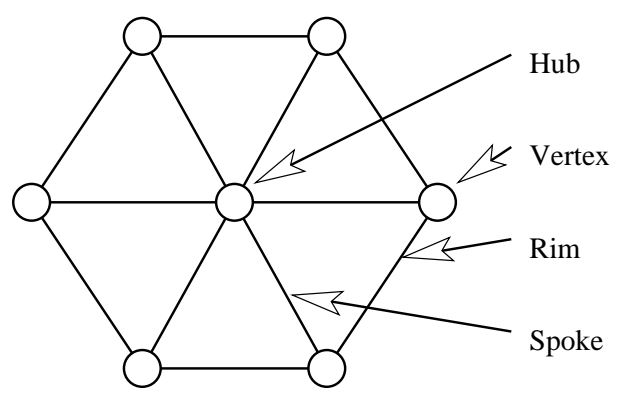

Fig. 3. The wheel graph $W_{6}$ demonstrating the naming convention we adopt for the elements of a wheel.

\subsection{Wheels}

As the wheel $W_{n}$ consists of a cycle $C_{n}$ together with a dominating hub vertex, $W_{n}$ has $n+1$ vertices and $2 n$ edges. The vertices $v_{1}$ through $v_{n}$ refer to the vertices of the cycle, with the rim edges $r_{1}$ through $r_{n}$ corresponding to the cycle edges $e_{1}$ through $e_{n}$. The spoke edges are those which connect the hub to a cycle vertex, and are given by $s_{i}=\left\{h u b, v_{i}\right\}$ for $1 \leq i \leq n$. We demonstrate this naming scheme graphically in Figure 3.

A general conjecture on VMTLs is that having vertices in a graph which differ widely with regard to their degrees prevents that graph from having a vertexmagic total labeling. This holds for wheels, which have a high-degree hub, as shown by MacDougall, Miller and Wallis in [3].

MacDougall et al. give two different methods of computing a feasible range for wheel graphs, and the true feasible range is given by the most restrictive maximum and minimum values. In addition to the bounds on the feasible range given earlier, the feasible range on wheels can been further bounded from below by $k \geq \frac{(n+1)(n+2)}{2}$ and above by $k \leq 7 n+6$ once you take the structure of the wheel into account. For the wheels $W_{n}$ with $n>11$, the minimum magic constant is larger than the maximum magic constant, so no VMTL can exist. MacDougall et al. also enumerate the VMTLs on wheels for $W_{3}$ (which is also the complete graph $\left.K_{4}\right), W_{4}$, and $W_{5}$ [3]. We extend these results, counting $W_{6}$ through $W_{10}$.

\section{Cycle Algorithm}

A naïve method to generate all vertex-magic total labelings for a graph is to simply try all $(|V|+|E|)$ ! permutations of the mapping of the labels onto the elements of the graph, and check to see if each result is a VMTL. Not only does this rapidly become infeasible on its own, as the size of the cycle increases it will also allow isomorphic copies of the same labeling to be generated independently. As such, every successfully generated VMTL must be compared to every other previously generated VMTL in order to remove duplicate copies. 


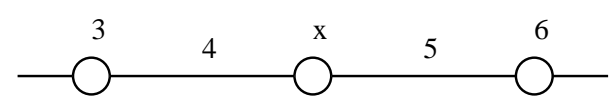

Fig. 4. A partial labeling of a piece of a graph with determined label $x$.

Our general approach is to apply vertex and edge labels working iteratively around the cycle. In the cycle VMTL generation algorithm, we remove cases of rotational symmetry by assigning the smallest vertex label to $v_{1}$, and then handle the reflective symmetry by making sure that $\lambda\left(e_{1}\right)<\lambda\left(e_{n}\right)$. Since $v_{1}$ must receive the smallest vertex label, it cannot be larger than $n+1$ or there will be insufficient labels to label the remaining vertices.

Since we are interested in calculating the number of VMTLs for each magic constant, the algorithm we develop takes both $n$ (the size of the cycle) and $k$ (the magic constant) as input.

We say that a label is a determined label if it contributes its value to the weight of a vertex for which every other contributing label is known. Assuming we know the magic constant we are trying to reach, there is only one possible value for the determined label. There are three conditions on a determined label $\lambda(x)$ which allow us to terminate the recursion tree at this node and backtrack. These conditions are:

1. $\lambda(x)<1$,

2. $\lambda(x)>|V|+|E|$, and

3. $\lambda(x)$ has already been used in this labeling.

Figure 4 gives a partial labeling and illustrates a determined label. In this example, if the desired magic constant is 20 , then $x$ must be 11 . However, if the desired magic constant is 15 , then $x$ would have to be 6 . Since 6 has already been used in this labeling, it would not be a valid partial labeling for $k=15$. If in a cycle we have magic constant $k$, then $\lambda\left(e_{2}\right)=k-\lambda\left(v_{2}\right)-\lambda\left(e_{1}\right)$. Then, once we know $\lambda\left(v_{3}\right)$ and $\lambda\left(e_{2}\right)$, we are able to determine $\lambda\left(e_{3}\right)$.

Our algorithm aims to obtain determined labels as quickly as possible. If a given label being applied in the algorithm is determined and the partial labeling is infeasible, then the entire computation subtree rooted at that partial labeling can immediately be excluded. Even in the worst-case scenario, where none of the determined labels eliminates a partial labeling, the use of a determined label reduces the branching factor at a position in the computation tree from $1 \leq i \leq|V|+|E|$ to 1 .

Before the algorithm itself is called, the global variables $n$ and $k$ are set with the size of the cycle and desired magic constant respectively, and the available list is initialized to every label being currently available. The actual algorithm begins with an initialization phase (by a call to initializeCycle()) which sets the labels of a vertex and two edges $\left(v_{1}, e_{1}\right.$, and $\left.e_{n}\right)$. The initialize function then calls extendCycle(2) which recursively labels the remaining vertices and edges. Execution completes when there is only one vertex $\left(v_{n}\right)$ remaining without 


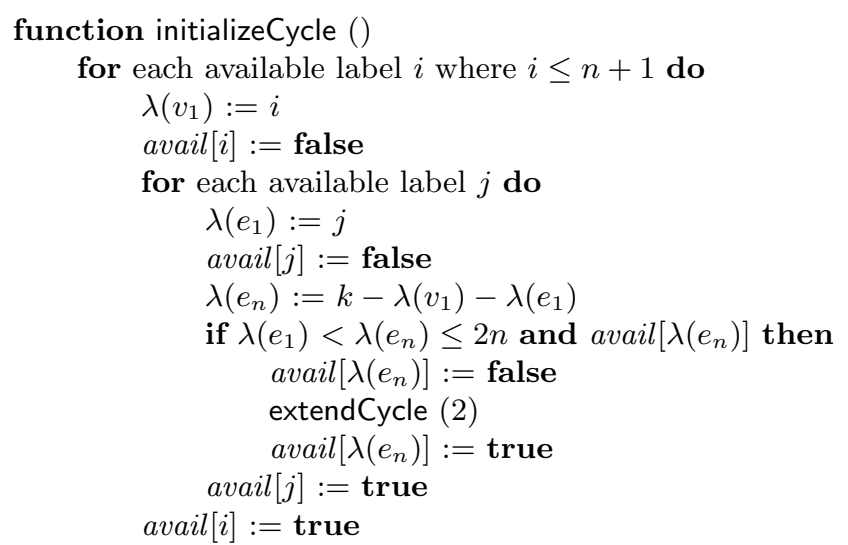

Fig. 5. Pseudocode for the initialization function for cycles. Global variables $n$ and $k$ are set to the desired values before the initializeCycle function is called.

a label. A linked list of unused labels is maintained at all times. This way, the more complete the partial labeling becomes, the fewer potential labels must be considered for each non-determined element.

The initialization function starts the labeling by attempting every possible label for vertex $v_{1}$ and edge $e_{1}$. We require that $\lambda\left(v_{1}\right)$ be the minimal vertex label in order to remove rotational symmetry. The maximum possible label for $v_{1}$ is $n+1$ due to the fact that since $v_{1}$ receives the minimum vertex label, we must retain $n-1$ labels greater than $\lambda\left(v_{1}\right)$ for the other vertices. This determines the label for edge $e_{n}$. In order to remove reflective symmetry, we require $\lambda\left(e_{n}\right)>$ $\lambda\left(e_{1}\right)$. The initialization function then calls the extend function with parameter 2. The pseudocode for the initialization function can be found in Figure 5.

The extend method takes a single parameter - the position $(t)$ in the cycle which is to be generated. A single loop applies, in turn, every unused label greater than $\lambda\left(v_{1}\right)$ to vertex $v_{t}$. Applying a label to $v_{t}$ determines the label for $e_{t}$. The extend method then calls itself with parameter $t+1$. The recursion terminates when $t=n$. At this point there is only one unlabeled element, $v_{n}$, which is obviously determined. If the single remaining label is the required label, then the VMTL is successfully completed and the Print() method is called. Print() is a generic function which can be used to perform any operation on the completed VMTL. In the case of enumeration, a count of the number of VMTLs is incremented. Figure 6 gives the pseudo-code for the extend function.

In order to obtain results more quickly, the algorithm is parallelized to run on multiple different processors. Each process is given an integer value as a command-line argument which acts as a static value for the first element to be assigned a label. Instead of iterating through all available values, the algorithm simply uses the supplied label. As the runtime increases for larger graphs, the 


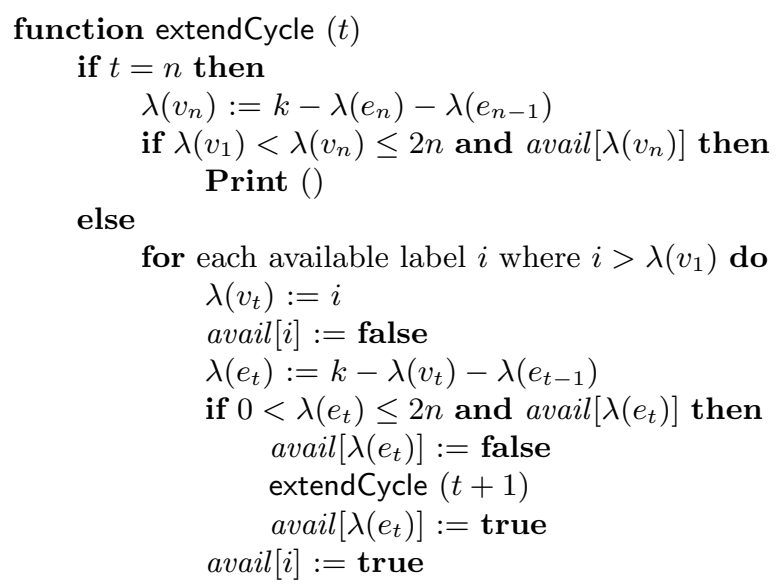

Fig. 6. Pseudocode for the extend function for cycles. Global variables $n$ and $k$ are set to the desired values before the extendCycle function is called.

Table 1. The total number of non-isomorphic VMTLs for cycle graphs $C_{n}(3 \leq n \leq$ $18)$.

\begin{tabular}{|r|r||r|r||r|r||r|r|}
\hline \multicolumn{7}{|c|}{$C_{n}$} \\
\hline \hline$n$ & Unique VMTLs & $n$ & Unique VMTLs & $n$ & Unique VMTLs & $n$ & Unique VMTLs \\
\hline 3 & 4 & 7 & 118 & 11 & 36128 & 15 & 74931690 \\
\hline 4 & 6 & 8 & 282 & 12 & 206848 & 16 & 613296028 \\
\hline 5 & 6 & 9 & 1540 & 13 & 1439500 & 17 & 5263250382 \\
\hline 6 & 20 & 10 & 7092 & 14 & 10066876 & 18 & 47965088850 \\
\hline
\end{tabular}

problem is distributed to more processors by supplying two seed values which determine the first two elements to receive labels.

\subsection{Results}

Table 1 gives the total number of EMTLs/VMTLs on the cycles $C_{3}$ through $C_{18}$, of which $C_{11}$ through $C_{18}$ had not previously been enumerated. Table 2 give the number of unique labelings broken down by magic constant.

\section{Wheel Algorithm}

Wheel graphs have a clear relationship to the cycles so the algorithm for generating all unique VMTLs on wheel $W_{n}$ bears a similarity to the algorithm for cycle $C_{n}$. However, the extra vertex and additional $n$ edges complicate the process. 
Table 2. The number of unique VMTLs for cycle graphs $C_{3}$ through $C_{10}$ broken down by magic constant $(k)$. (Note that the duals have not been included.)

\begin{tabular}{|c|c|c|c|c|c|c|c|}
\hline \multicolumn{2}{|r|}{$C_{3}$} & \multicolumn{2}{|r|}{$C_{4}$} & \multicolumn{2}{|r|}{$C_{5}$} & \multicolumn{2}{|r|}{$C_{6}$} \\
\hline$k$ & Unique VMTLs & $k$ & Unique VMTLs & $k$ & Unique VMTLs & $k$ & Unique VMTLs \\
\hline 9 & 1 & 12 & 1 & 14 & 1 & 17 & 3 \\
\hline 10 & 1 & 13 & 2 & 15 & 0 & 18 & 1 \\
\hline & & & & 16 & 2 & 19 & 6 \\
\hline
\end{tabular}

\begin{tabular}{|l|r||r|r|r|r|||r|r|}
\hline \multicolumn{2}{|c||}{$C_{7}$} & \multicolumn{2}{c|}{$C_{8}$} & \multicolumn{2}{c||}{$C_{9}$} & \multicolumn{2}{c|}{$C_{10}$} \\
\hline \hline$k$ & Unique VMTLs & $k$ & Unique VMTLs & $k$ & Unique VMTLs & $k$ & Unique VMTLs \\
\hline 19 & 9 & 22 & 10 & 24 & 31 & 27 & 125 \\
\hline 20 & 10 & 23 & 19 & 25 & 43 & 28 & 236 \\
\hline 21 & 11 & 24 & 57 & 26 & 125 & 29 & 698 \\
\hline 22 & 29 & 25 & 55 & 27 & 264 & 30 & 1138 \\
\hline & & & & 28 & 307 & 31 & 1349 \\
\hline
\end{tabular}

\begin{tabular}{|c|c|c|c|c|c|c|c|}
\hline \multicolumn{2}{|r|}{$C_{11}$} & \multicolumn{2}{|r|}{$C_{12}$} & \multicolumn{2}{|r|}{$C_{13}$} & \multicolumn{2}{|r|}{$C_{14}$} \\
\hline$k$ & Unique VMTLs & $k$ & Unique VMTLs & $k$ & Unique VMTLs & $k$ & Unique VMTLs \\
\hline 29 & 308 & 32 & 1602 & 34 & 3809 & 37 & 32077 \\
\hline 30 & 711 & 33 & 4111 & 35 & 10967 & 38 & 91866 \\
\hline 31 & 1781 & 34 & 10834 & 36 & 33951 & 39 & 299525 \\
\hline 32 & 3371 & 35 & 19183 & 37 & 79234 & 40 & 576701 \\
\hline 33 & 4945 & 36 & 30877 & 38 & 139499 & 41 & 977354 \\
\hline 34 & 6948 & 37 & 36817 & 39 & 202253 & 42 & 1427929 \\
\hline & & & & 40 & 250037 & 43 & 1627986 \\
\hline
\end{tabular}

\begin{tabular}{|c|c|c|c|c|c|c|c|}
\hline \multicolumn{2}{|r|}{$C_{15}$} & \multicolumn{2}{|r|}{$C_{16}$} & \multicolumn{2}{|r|}{$C_{17}$} & \multicolumn{2}{|r|}{$C_{18}$} \\
\hline$k$ & Unique VMTLs & $k$ & Unique VMTLs & $k$ & Unique VMTLs & $k$ & Unique VMTLs \\
\hline 39 & 63995 & 42 & 884789 & 44 & 1152784 & 47 & 26677502 \\
\hline 40 & 284590 & 43 & 2706053 & 45 & 8660408 & 48 & 104169715 \\
\hline 41 & 889063 & 44 & 8685625 & 46 & 30280605 & 49 & 351608789 \\
\hline 42 & 2332807 & 45 & 20266824 & 47 & 86881643 & 50 & 859974262 \\
\hline 43 & 4402572 & 46 & 37574150 & 48 & 187828262 & 51 & 1815449072 \\
\hline 44 & 7339913 & 47 & 59829497 & 49 & 336981439 & 52 & 3082588134 \\
\hline 45 & 10395599 & 48 & 83018416 & 50 & 511013242 & 53 & 4648495519 \\
\hline 46 & 11757306 & 49 & 93682660 & 51 & 683131331 & 54 & 6154283390 \\
\hline & & & & 52 & 785695477 & 55 & 6939298042 \\
\hline
\end{tabular}


As with the cycle algorithm, our wheel VMTL generation algorithm applies vertex and edge labels working iteratively around the edge of the cycle portion of the wheel. We remove rotational symmetry by assigning the smallest spoke label to $s_{1}$. As with cycles, reflective symmetry is removed by ensuring that $\lambda\left(r_{1}\right)<\lambda\left(r_{n}\right)$. We use the $s_{1}$ instead of the $v_{1}$ to remove rotational symmetry for the wheel in order to trim the computation tree of partial labelings which will result in a hub with excessive weight more efficiently. Since $s_{1}$ must receive the smallest spoke label, it cannot be larger than $2 n+2$ or there will be insufficient labels to label the remaining spokes.

Determined labels continue to be an asset to remove subtrees of the computation tree. In this case, we require three labels in order to determine a fourth. For example, $\lambda\left(r_{2}\right)=k-\lambda\left(s_{2}\right)-\lambda\left(v_{2}\right)-\lambda\left(r_{1}\right)$.

As in the case of the cycles, before the algorithm itself is called, the global variables $n$ and $k$ are set with the size of the wheel and desired magic constant respectively, and the available list is initialized to every label being currently available. The actual algorithm begins with an initialization phase (by a call to initializeWheel ()$)$ which labels $s_{1}, e_{1}, e_{n}$, and $v_{1}$ in such a way as to prevent isomorphic labelings from being generated. The initialize function then calls extendWheel(2) which recursively labels a spoke, exterior vertex, and rim and calls itself until only $s_{n}, v_{n}$, and the hub remain, which are then labeled by a call to finalizeWheel(). Also like the cycle algorithm, a linked list consisting of the unused labels is maintained in order to improve efficiency as the partial labeling becomes more complete.

The initialization function starts the labeling by attempting every possible label for spoke edge $s_{1}$ and rim edge $r_{1}$. Every possible label greater than $\lambda\left(r_{1}\right)$ is applied to $r_{n}$, thus removing reflective symmetry. This determines the label for vertex $v_{1}$. The initialization function then calls the extend function with parameter 2. The pseudocode for the initialization function is given in Figure 7.

The extend function takes a single parameter $t$ which gives the position of the wheel currently being expanded and applies every possible label greater than $\lambda\left(s_{1}\right)$ to $s_{t}$. The extend function then applies every possible label to $v_{t}$ which determines the label for $r_{t}$. The finalize function is called when $t=n$.

In order to prune the computation tree more effectively, we keep a close watch on the weight of the hub vertex through the variable $h u b W$ eight. Due to its high degree, its weight can easily exceed the desired magic constant. Every time a label is applied to a spoke, the partial hub weight variable is updated. Once in each iteration of the extend method, we check to ensure that the minimum weight the hub can achieve is less than or equal to the desired magic constant. The minimal weight is given by the partial weight plus the smallest unused label (applied to the hub) and the $n-t$ smallest unused labels which are greater than $\lambda\left(s_{1}\right)$. If the minimal hub weight is larger than the desired magic constant, the partial labeling fails and the next set of labels is considered. The pseudocode for the extend function can be found in Figure 8.

The finalize function tries every available label for $s_{n}$ which is greater than $\lambda\left(s_{1}\right)$. This determines the labels for both $v_{n}$ and the hub. If these last labels 


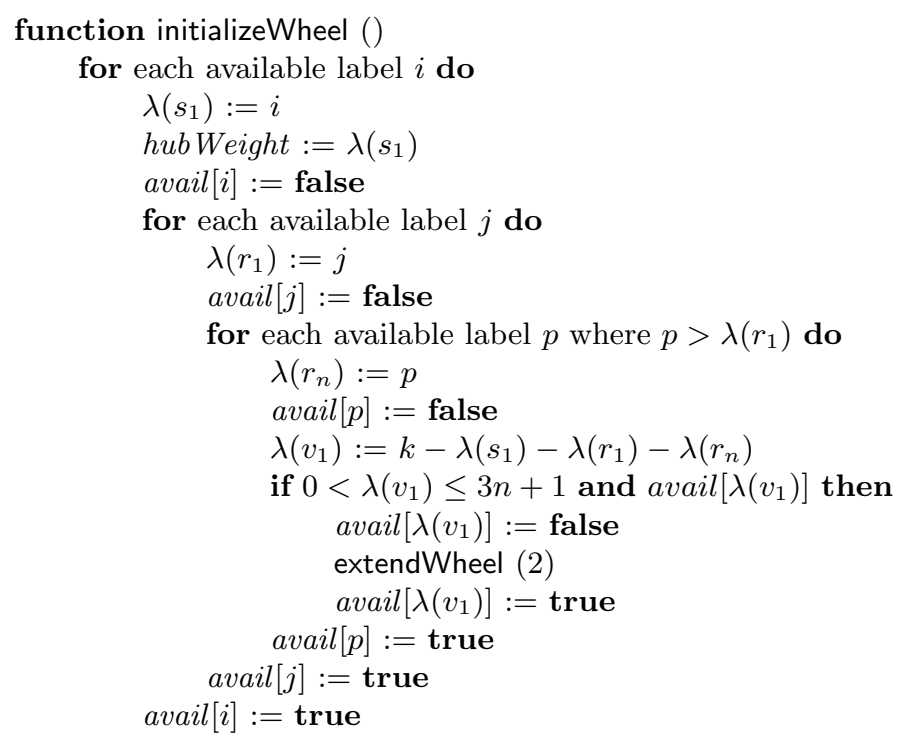

Fig. 7. Pseudocode for the initialization function for wheels. Global variables $n$ and $k$ are set to the desired values before the initializeWheel function is called.

can be successfully applied, then the Print() method is called which increments the number of labelings. Figure 9 gives the pseudocode for the finalize function.

\subsection{Results}

Table 3 gives the total number of VMTLs on the wheels $W_{3}$ through $W_{10}$, of which $W_{6}$ through $W_{10}$ had not previously been enumerated. Results on $W_{11}$ are currently pending completion. Table 4 gives the number of unique labelings broken down by magic constant. Of particular note is the fact that $W_{9}$ does not have a VMTL for $k=58$ even though it is in the feasible range as given by MacDougall, Miller, and Wallis in [3]. Goemans gave a counting argument showing why no VMTL on $W_{9}$ can have $k=58$ after we posed the problem of the missing labeling [6].

\section{Conclusion and Open Problems}

As there are EMTLs/VMTLs for all cycles $C_{n}$ with $n \geq 3$, the number of unique labelings on larger cycles remain an open problem. It is desirable, however, to determine a formula which gives the number of EMTLs/VMTLs on a cycle of size $n$ without having to actually count them.

In addition to the wheels, MacDougall, Miller and Wallis present other related classes of graphs which have similar size restrictions [3]. Figure 10 gives 


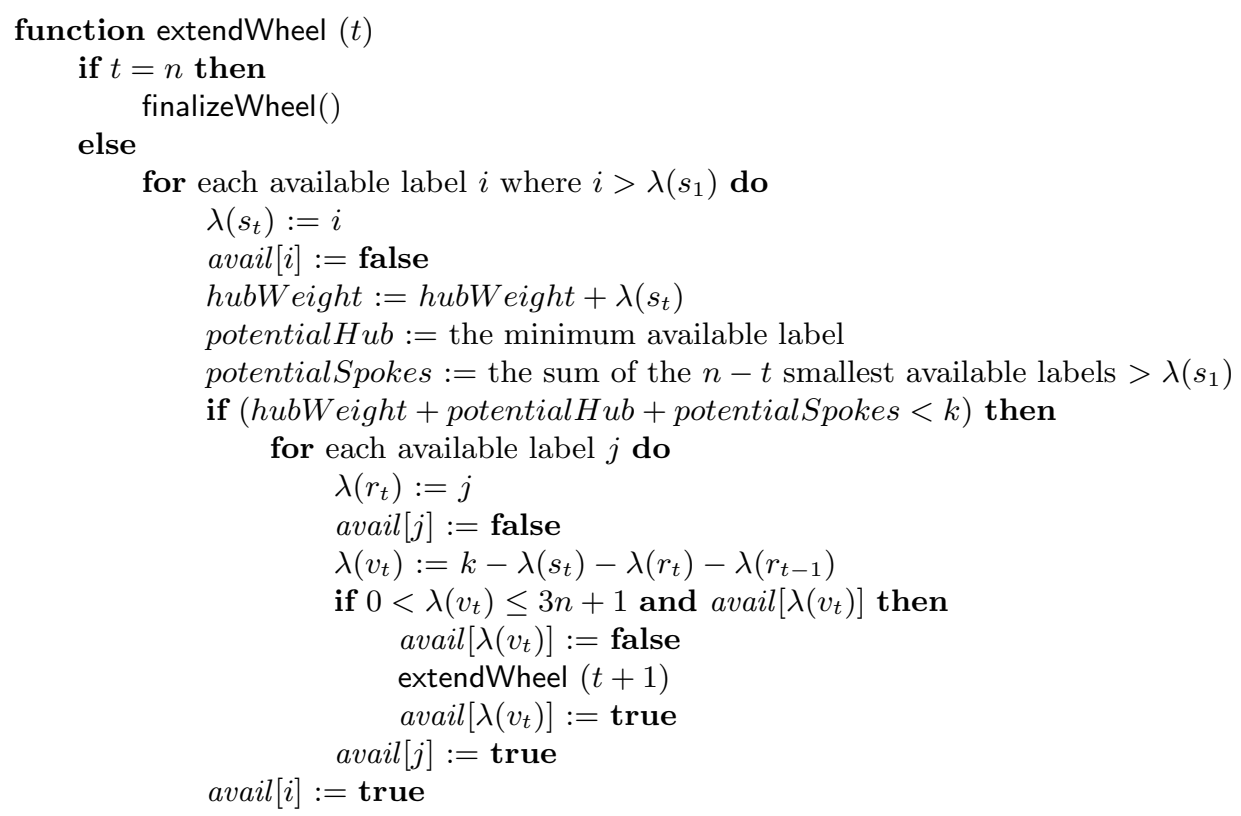

Fig. 8. Pseudocode for the extend function for wheels. Global variables $n$ and $k$ are set to the desired values before the extendWheel function is called.

sample graphs for three of these related classes: fans, $t$-fold wheels, and friendship graphs.

\section{Acknowledgements}

This work was made possible by the facilities of the Shared Hierarchical Academic Research Computing Network (SHARCNET:www.sharcnet.ca).

\section{References}

1. Wallis, W.D.: Magic Graphs. Birkhäuser, New York, NY, USA (2001)

2. Gallian, J.A.: A dynamic survey of graph labeling. The Electronic Journal of Combinatorics 15 (2008) \#DS6

3. MacDougall, J.A., Miller, M., Wallis, W.D.: Vertex-magic total labelings of wheels and related graphs. Utilitas Mathematica 62 (2002) 175-183

4. MacDougall, J.A., Miller, M., Slamin, Wallis, W.D.: Vertex-magic total labelings of graphs. Utilitas Mathematica 61 (2002) 3-21

5. Godbold, R.D., Slater, P.J.: All cycles are edge-magic. Bulletin of the ICA 22 (1998) 93-97

6. Goemans, M. personal communication (2008) 


\section{function finalizeWheel ()}

for each available label $i$ where $i>\lambda\left(s_{1}\right)$ do

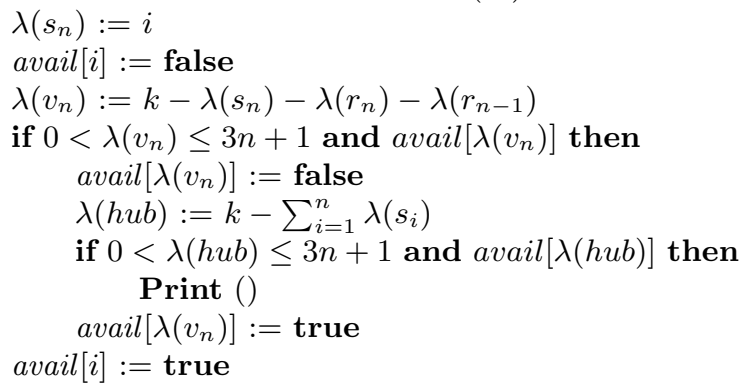

Fig. 9. Pseudocode for the finalize function for wheels. Global variables $n$ and $k$ are set to the desired values before the finalizeWheel function is called.

Table 3. The total number of non-isomorphic VMTLs for wheel graphs $W_{n}(3 \leq n \leq$ 8).

\begin{tabular}{|r|r||r|r||l|r|}
\hline \multicolumn{5}{|c|}{$W_{n}$} \\
\hline \hline$n$ & Unique VMTLs & $n$ & Unique VMTLs & $n$ & Unique VMTLs \\
\hline 3 & 14 & 6 & 859404 & 9 & 17804388662 \\
\hline 4 & 2080 & 7 & 22063500 & 10 & 418858095690 \\
\hline 5 & 31892 & 8 & 637402504 & 11 & pending \\
\hline
\end{tabular}
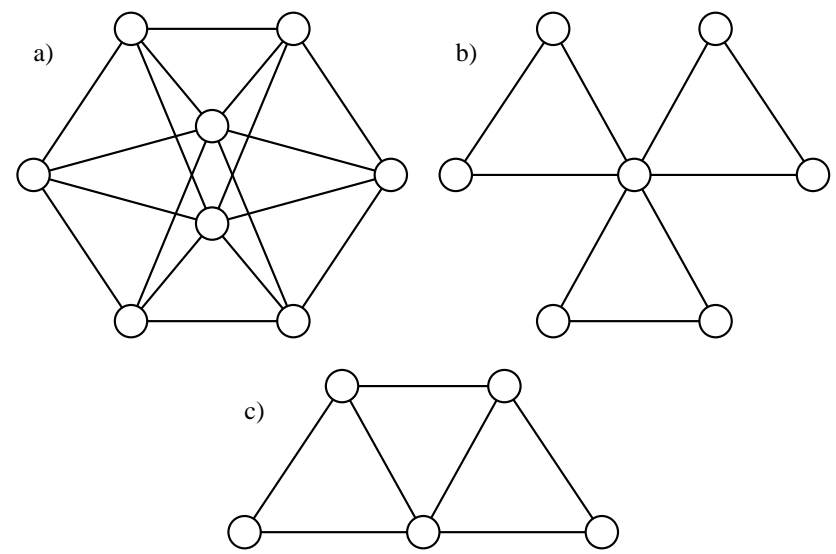

Fig. 10. Examples of graphs in three classes related to the wheels: a) $t$-fold wheel, b) friendship graph, c) fan. 
Table 4. The number of unique VMTLs for wheel graphs $W_{3}$ through $W_{5}$ broken down by magic constant $(k)$.

\begin{tabular}{|l|r||r|r||r|r|}
\hline \multicolumn{2}{|c|}{$W_{3}$} & \multicolumn{2}{|c|}{$W_{4}$} & \multicolumn{2}{|c|}{$W_{5}$} \\
\hline \hline$k$ & Unique VMTLs & $k$ & Unique VMTLs & $k$ & Unique VMTLs \\
\hline 19 & 0 & 26 & 89 & 32 & 239 \\
\hline 20 & 2 & 27 & 149 & 33 & 1242 \\
\hline 21 & 5 & 28 & 522 & 34 & 2694 \\
\hline 22 & 0 & 29 & 376 & 35 & 5180 \\
\hline 23 & 5 & 30 & 573 & 36 & 7873 \\
\hline 24 & 2 & 31 & 211 & 37 & 7173 \\
\hline 25 & 0 & 32 & 131 & 38 & 4124 \\
\hline & & 33 & 29 & 39 & 2511 \\
\hline & & & & 40 & 776 \\
\hline & & & & 41 & 80 \\
\hline
\end{tabular}

\begin{tabular}{|l|r||r|r||r|r|}
\hline \multicolumn{2}{|c|}{$W_{6}$} & \multicolumn{2}{c||}{$W_{7}$} & \multicolumn{1}{c|}{$W_{8}$} \\
\hline \hline$k$ & Unique VMTLs & $k$ & Unique VMTLs & $k$ & Unique VMTLs \\
\hline 39 & 5978 & 45 & 24998 & 52 & 795294 \\
\hline 40 & 36945 & 46 & 204170 & 53 & 7352502 \\
\hline 41 & 76335 & 47 & 880257 & 54 & 28521585 \\
\hline 42 & 158805 & 48 & 2198247 & 55 & 64090384 \\
\hline 43 & 173887 & 49 & 3637665 & 56 & 106131735 \\
\hline 44 & 187409 & 50 & 4760707 & 57 & 132239986 \\
\hline 45 & 116447 & 51 & 4425875 & 58 & 133415487 \\
\hline 46 & 77827 & 52 & 3384967 & 59 & 92798616 \\
\hline 47 & 21793 & 53 & 1818749 & 60 & 53134373 \\
\hline 48 & 3978 & 54 & 646233 & 61 & 17008206 \\
\hline & & 55 & 81632 & 62 & 1914336 \\
\hline
\end{tabular}

\begin{tabular}{|l|r||r|r||r|r|}
\hline \multicolumn{2}{|c|}{$W_{9}$} & \multicolumn{2}{c||}{$W_{10}$} & \multicolumn{2}{|c|}{$W_{11}$} \\
\hline \hline$k$ & Unique VMTLs & $k$ & Unique VMTLs & $k$ & Unique VMTLs \\
\hline 58 & 0 & 66 & 1739667155 & 78 & pending \\
\hline 59 & 34364364 & 67 & 4780216858 & 79 & pending \\
\hline 60 & 236314351 & 68 & 18515045434 & 80 & pending \\
\hline 61 & 833847423 & 69 & 39874554946 & 81 & pending \\
\hline 62 & 1846542901 & 70 & 75518840087 & 82 & 162942689359 \\
\hline 63 & 2996328931 & 71 & 84888911188 & 83 & 8201853531 \\
\hline 64 & 3821193834 & 72 & 90187289669 & & \\
\hline 65 & 3553033163 & 73 & 60230503071 & & \\
\hline 66 & 2649033979 & 74 & 33425583234 & & \\
\hline 67 & 1364327018 & 75 & 9122758622 & & \\
\hline 68 & 435740211 & 76 & 574725426 & & \\
\hline 69 & 33662487 & & & & \\
\hline
\end{tabular}

